\title{
THE SET OF CHARACTER DEGREES OF A FINITE GROUP DOES NOT DETERMINE ITS SOLVABILITY
}

\author{
GABRIEL NAVARRO \\ (Communicated by Pham Huu Tiep)
}

\begin{abstract}
We find a pair of groups, one solvable and the other non-solvable, with the same set of character degrees.
\end{abstract}

\section{INTRODUCTION}

Despite the abundant literature on the set of character degrees of finite groups, a basic question has remained unsettled until now. Suppose that $G$ is a finite group, let $\operatorname{Irr}(G)$ be the set of the irreducible complex characters of $G$, and write

$$
\operatorname{cd}(G)=\{\chi(1) \mid \chi \in \operatorname{Irr}(G)\}
$$

for the set of character degrees of $G$.

Theorem A. There exist two finite groups $G$ and $H$ such that $\operatorname{cd}(G)=\operatorname{cd}(H)$, with $G$ solvable and $H$ non-solvable.

This solves Problem (8.4) in [3, for instance. The non-solvable group $H$ in Theorem A is not simple. In fact, B. Huppert has conjectured that if $H$ is simple and $\operatorname{cd}(G)=\operatorname{cd}(H)$, then $G=H \times A$ for some abelian group $A$ [2]. Theorem A is also closely related to R. Brauer's Problem 2 in [1. Brauer asked what kind of properties the group algebra $\mathbb{C} G$ knows about the group $G$. Even if we now know that $\operatorname{cd}(G)$ does not determine the solvability of $G$, perhaps the group algebra does.

\section{The Groups}

For our non-solvable group, we let $H$ be the perfect group of order $37500=$ $2^{2} \cdot 3 \cdot 5^{5}$, which is an extension of the alternating group $A_{5}$ of degree 5 by an elementary abelian 5 -group of order $5^{4}$. This is a group with set of character degrees $\mathcal{A}=\{1,3,4,5,10,12,15,20,30,60\}$.

Now, we construct a solvable group $G$ such that $\operatorname{cd}(G)=\mathcal{A}$. Let $V=G F\left(3^{4}\right)$ be the additive group of the field of dimension 4 over $G F(3)$. Let $\sigma$ be a generator of the Galois group $\operatorname{Gal}\left(G F\left(3^{4}\right) / G F(3)\right)$ of order 4, let $U$ be the subgroup of order 5 inside the multiplicative group $G F\left(3^{4}\right)^{\times}$, and let $X=V D$ be the semidirect product of order $2^{2} \cdot 3^{4} \cdot 5$, where $D=U\langle\sigma\rangle$. The action of $D$ on $\operatorname{Irr}(V)$ has 8 orbits with representatives $\mathcal{B}=\left\{1_{V}, \lambda_{i}, \epsilon_{j}, \rho_{k}\right\}, i=1,2, j=1,2$, and $k=1,2,3$. The $D$-orbit of $\lambda_{i}$ has size 5 for $i=1,2$; the $D$-orbit of $\epsilon_{j}$ has size 20 for $j=1,2$;

Received by the editors July 3, 2013.

2010 Mathematics Subject Classification. Primary 20C15.

Key words and phrases. Character degrees, solvability, finite groups.

The authors research was partially supported by the Spanish Ministerio de Educación y Ciencia, proyecto MTM2010-15296, and Prometeo/Generalitat Valenciana.

(C) 2014 American Mathematical Society Reverts to public domain 28 years from publication 
and the $D$-orbit of $\rho_{k}$ has size 10 for $k=1,2,3$. Every irreducible character of $V$ extends to its stabilizer. Hence, it is easy to check that $\operatorname{cd}(X)=\{1,4,5,10,20\}$.

Now, let $Y=A_{4}$, the alternating group of degree 4 . Hence $\operatorname{cd}(Y)=\{1,3\}$. We now let $G=X \times Y$, and we have that

$$
\operatorname{cd}(G)=\{n m \mid n \in \operatorname{cd}(X), m \in \operatorname{cd}(Y)\}=\mathcal{A} .
$$

\section{REFERENCES}

[1] Richard Brauer, Representations of finite groups, Lectures on Modern Mathematics, Vol. I, Wiley, New York, 1963, pp. 133-175. MR0178056 (31 \#2314)

[2] Bertram Huppert, Some simple groups which are determined by the set of their character degrees. I, Illinois J. Math. 44 (2000), no. 4, 828-842. MR.1804317 (2001k:20009)

[3] Gabriel Navarro, Problems in character theory, Character theory of finite groups, Contemp. Math., vol. 524, Amer. Math. Soc., Providence, RI, 2010, pp. 97-125, DOI 10.1090/conm/524/10350. MR2731923 (2012a:20014)

Departament d’Àlgebra, Universitat de València, 46100 Burjassot, València, Spain E-mail address: gabriel@uv.es 